\title{
Das Recht, nicht in Armut leben zu müssen und die Unterscheidung zwischen vollkommenen und unvollkommenen Pflichten
}

\author{
- Eine begriffliche Flurbereinigung -
}

\section{Hubert Schnüriger, Basel}

„Die weltweite Armut stellt eine moralische Herausforderung dar, die alle Menschen in die Pflicht nimmt." Auch wenn diese Aussage nicht gänzlich unumstritten ist, spiegelt sie in dieser allgemeinen Form einen breit abgestützten Konsens innerhalb der praktischen Philosophie wider. Sie wird hier denn auch nicht zur Diskussion gestellt. Umstritten ist hingegen, ob zugleich alle Menschen ein vorinstitutionelles moralisches Recht haben, nicht in Armut leben zu müssen. Gegen ein solches Recht werden gerne begriffliche Bedenken angeführt, die sich in gleicher Weise gegen alle Arten moralischer sozioökonomischer Rechte richten. Ihre paradigmatische Form finden diesen Bedenken im Einwand von Onora O'Neill, dass sozioökonomische Rechte Institutionen voraussetzten, welche festlegten, wer gegenüber wem ein solches Recht geltend machen könne. O`Neill verknüpft diesen Einwand mit der allgemeineren These, dass zwischen vollkommenen Pflichten, denen Rechte anderer korrelieren und unvollkommenen Pflichten, denen keine Rechte anderer korrelieren, zu unterscheiden sei.

Im ersten Kapitel wird der begriffliche Rahmen gelegt, um den Einwand von O'Neill im folgenden Kapitel in einer systematisch angemessenen Weise formulieren zu können. Das dritte Kapitel untersucht die These näher, dass unvollkommenen Pflichten keine Rechte korrelieren können. Diese These wird in der Regel über eine von drei Arten der Unterscheidung zwischen vollkommenen und unvollkommenen Pflichten eingeführt. Das Kapitel fragt, weshalb genau unvollkommenen Pflichten keine Rechte korrelieren können sollen. Wie zu zeigen versucht wird, scheint die These in allen Versionen einen problematischen Rechtsbegriff vorauszusetzen und entsprechend die Rolle von Rechten im praktischen Denken in einer spezifischen, keineswegs allgemein anerkannten Weise zu interpretieren. Im letzten Kapitel wird nicht nur die Sinnhaftigkeit der Rede von einem Recht eines jeden Menschen, nicht in Armut leben zu müssen vor dem Hintergrund eines plausiblen Rechtsbegriffs verteidigt, sondern auch darauf hingewiesen, dass über einen solchen Rechtsbegriff die Debatte über sozioökonomische Menschenrechte auf ein stabiles Fundament gestellt werden kann.

\section{Der begriffliche Rahmen}

Die Vorbehalte gegenüber dem Begriff eines vorinstitutionellen moralischen Rechts aller Menschen, nicht in Armut leben zu müssen, liegen in der triadische Struktur von Rechten begründet: Ein jedes Recht ist als das Recht eines Individuums, des Rechtssubjekts, gegenüber einem (anderen) moralischen Akteur, dem Rechtsadressaten, auf etwas zu verstehen. ${ }^{1}$ Gegenstand von Rechten sind diesem Strukturmodell gemäss grundsätzlich Handlungen oder Handlungsunterlassungen. Für die Frage nach der Möglichkeit sozioökonomischer Menschenrechte kommen nur Rechte in den Blick, welche Handlungen des Rechtsadressaten zum Gegenstand haben. In diesem Fall hat letzterer dem Rechtssubjekt gegenüber die Pflicht, die entsprechende Handlung auszuführen oder zu unterlassen. Es handelt sich um sogenannte Anspruchsrechte. Dieses Verhältnis von Rechten und Pflichten

\footnotetext{
${ }^{1}$ Vgl. Alexy, Theorie der Grundrechte, 1985, S. 171f.
} 
wird als die Korrelativität von Rechten und Pflichten bezeichnet. ${ }^{2}$ Jedem Recht korreliert in diesem Sinne eine Pflicht.

In dieser Tradition stellen zweigliedrige Wendungen wie ,das Recht, nicht in Armut leben zu müssen“ eine abkürzende Redeweise dar. Sie können als Kurzformen für ein ganzes Bündel von Rechten oder als Kurzformen für ein einzelnes Recht stehen. Wie der Inhalt des Rechts, nicht in Armut leben zu müssen genau zu bestimmen ist, hängt von substantiellen Überlegungen ab. Ein naheliegender Ansatz versteht den Ausdruck, das Recht, nicht in Armut leben zu müssen' als abkürzende Bezugnahme auf das Recht eines Rechtssubjekts gegenüber einem moralischen Akteur, dass dieser dem Rechtssubjekt hilft, nicht in Armut leben zu müssen. Diese Formulierung ist selbstverständlich nicht nur inhaltlich abstrakt, sondern sie lässt auch offen, wer Adressat des Rechts ist. Entscheidend für den Moment ist, dass das Recht wie alle sozioökonomischen Rechte eine positive Pflicht des Rechtsadressaten impliziert. Damit ist gemeint, dass es eine Pflicht ist, etwas zu tun und nicht nur, eine bestimmte Handlung zu unterlassen. Es ist gerade dieser positive Charakter der korrelativen Pflichten sozioökonomischer Rechte, welcher am Ursprung des hier zu untersuchenden Einwandes steht.

Um den Einwand angemessen verorten und formulieren zu können, muss zusätzlich zwischen verschiedenen Formen von Rechten unterschieden werden. Diese Unterscheidung bezieht sich auf formale Eigenschaften der Rechtssubjekte und der Rechtsadressaten. ${ }^{3}$ Rechte, die jedem Individuum innerhalb einer bestimmten Klasse zukommen lassen sich als universelle Rechte bezeichnen und von partikularen Rechten abgrenzen, die nur einem bestimmten Individuum oder bestimmten Individuen zukommen. Menschenrechte gelten als paradigmatische Beispiele für universelle Rechte, da sie jedem Menschen zukommen. Generelle Rechte sind Rechte, die gegenüber jedem moralischen Akteur einer bestimmten Klasse bestehen. Spezielle Rechte bestehen hingegen nur gegenüber bestimmten moralischen Akteuren. Auch hier ist im Blick zu behalten, dass Rechte im engeren Sinne eine Beziehung zwischen zwei Individuen zum Ausdruck bringen. Der Ausdruck ,universelles und generelles Recht, nicht in Armut leben zu müssen' stellt entsprechend eine abkürzende Bezugnahme auf ein ganzes Bündel von Rechten dar: Jedes Individuum hat gegenüber jedem moralischen Akteur jeweils ein Recht, dass dieser ihm hilft, nicht in Armut leben zu müssen. Wenn dieser Sachverhalt im Blick behalten wird, kann der einfacheren sprachlichen Praxis gefolgt werden.

Diese einleitenden Bemerkungen lassen offen, ob es sich beim Recht, nicht in Armut leben zu müssen um ein Menschenrecht handelt. $\mathrm{Ob}$ dem so ist, hängt davon $\mathrm{ab}$, wie der Begriff der Menschenrechte bestimmt wird. Grob lässt sich zwischen zwei Positionen unterscheiden. Eine erste bestimmt Menschenrechte über eine spezifisch politische Rolle, die ihnen zukommen soll. ${ }^{4}$ Als Ausgangspunkt für dieses Verständnis gilt die Menschenrechtspraxis, wie sie sich in der internationalen Politik und im Völkerrecht darstellt. Eine zweite Position bestimmt Menschenrechte als moralische Rechte, die jedem Menschen aufgrund seines Menschseins zukommen. Diesem Verständnis gemäss stellen Menschenrechte eine besondere Art von moralischen oder natürlichen Rechten dar. ${ }^{5}$ Damit ist nicht nur gemeint, dass sie vor und unabhängig von konventionellen, rechtlichen oder politischen Institutionalisierungen bestehen, sondern auch, dass ihre Existenz und inhaltliche Bestimmung grundsätzlich in den Bereich des allgemeinen moralischen Denkens gehören. ${ }^{6}$ Um Missverständnisse auszuschliessen, muss hier allerdings zwischen zwei Lesarten von

\footnotetext{
${ }^{2}$ Vgl. Hohfeld, Some Fundamental Legal Conceptions as Applied in Judicial Reasoning, Yale L. J. 23 (1913), S. $16(31 \mathrm{f})$.

${ }^{3}$ Vgl. Koller, Theorie des Rechts. Eine Einführung, 2., verb. und erw. Aufl. 1997, S. 96ff.

${ }^{4}$ Vgl. z. B. Rawls, The Law of Peoples, 1999, S. 79ff; Beitz, The Idea of Human Rights, 2009, S. $102 \mathrm{ff}$.

${ }^{5}$ Vgl. z. B. Sen, Elements of a Theory of Human Rights, Philos. Public Aff. 32/4 (2004), S. 315 (320ff., 345).

${ }^{6}$ Vgl. Tasioulas, On the Nature of Human Rights, in: Ernst/Heilinger (Hrsg.), The Philosophy of Human Rights. Contemporary Controversies, 2012, S. 17 (26f.).
} 
Menschenrechten als moralischen Rechten unterschieden werden. Entsprechend einer ersten Lesart hängt die Existenz von Menschenrechten zwar nicht davon ab, dass sie bereits in der einen oder anderen Form institutionalisiert und damit sozial anerkannt sind, sie sind aber auf ihre Institutionalisierung hin angelegt. Das wird oft mit der Vorstellung verknüpft, dass Menschenrechte qua Rechte in den Bereich der Gerechtigkeit gehören. ${ }^{7}$ Die zweite Lesart bindet weder den Begriff von Menschenrechten noch jenen von Rechten überhaupt an den Bereich der Gerechtigkeit. Dieser Lesart gemäss hat der Begriff eines moralischen Rechts wie jener der moralischen Pflicht seinen Platz sowohl im Kontext institutionenethischer als auch im Kontext individualethischer Annäherungen an das Problem der Weltarmut. Hintergrund dieser unterschiedlichen Lesarten ist die Unterscheidung zwischen Fragen der Gerechtigkeit als Fragen der gerechten Einrichtung von sozialen Institutionen und Fragen der nichtinstitutionellen, individuellen Moral. $^{8}$

Ohne dies im Einzelnen an dieser Stelle zu begründen, ${ }^{9}$ wird diesem Aufsatz ein Verständnis von Menschenrechten als universellen moralischen Rechten zugrunde gelegt. Dies bietet sich alleine schon aus Gründen der sprachlichen Einfachheit an. Für die weiteren Ausführungen ist diese Festlegung denn auch nicht entscheidend, da durch einen politischen Menschenrechtsbegriff nicht bereits der Begriff eines moralischen Rechts ausgeschlossen wird, das jedem Menschen aufgrund seines Menschseins zukommt. Die Auseinandersetzung über die begriffliche Möglichkeit eines universellen moralischen Rechts, nicht in Armut leben $\mathrm{zu}$ müssen, lässt sich entsprechend unabhängig davon führen, ob es sich dabei um ein Menschenrecht handelt. Gleichzeitig wird zunächst offen gelassen, ob der Rechtsbegriff ausschliesslich in den Bereich institutionenethischer Problemstellungen gehört. Einige der Beispiel im dritten Kapitel sowie die Ausführungen im letzten Teil machen jedoch deutlich, dass es sich nahelegt, den Rechtsbegriff in einem weiteren Sinne zu verstehen, der Rechten sowohl in institutionen- als auch in individualethischen Zusammenhängen eine zentrale Rolle zukommen lässt.

Aufbauend auf diesen terminologischen Unterscheidungen lässt sich der Vorbehalt gegenüber sozioökonomischen Menschenrechten präzise fassen. Kritiker des Begriffs sozioökonomischer Menschenrechte bestreiten, dass es universelle und generelle moralische Rechte auf positive Handlungen geben kann. Sozioökonomische Rechte könne es nur gegenüber spezifischen Akteuren geben. Um aber bestimmen zu können, welchen spezifischen Akteuren gegenüber die Rechte bestehen, bedürfe es einer institutionellen Zuordnung. Mithin könne es keine vorinstitutionellen sozioökonomischen Menschenrechte geben. Die einflussreichste Formulierung dieses Einwandes stammt, wie bereits erwähnt, von Onora O’Neill.

\section{Der paradigmatische Vorbehalt gegenüber generellen sozioökonomischen Rechten}

O’Neills Einwand gegen den Begriff sozioökonomischer Menschenrechte lässt sich über drei Schritte rekonstruieren. ${ }^{10}$ Erstens setzt O'Neill voraus, dass (Anspruchs-)Rechte gegenüber identifizierbaren anderen geltend gemacht oder in Anspruch genommen werden können müssen. Zweitens geht sie davon aus, dass sozioökonomische Rechte nicht gegenüber allen moralischen Akteuren geltend gemacht werden können. Daraus schliesst sie drittens, dass es keine generellen sozioökonomischen Menschenrechte geben kann. O’Neill will damit nicht

\footnotetext{
7 So z. B. Ashford, Obligations of Justice and Beneficence to Aid the Severely Poor, in: Illingworth/Pogge/Wenar (Hrsg.), Giving Well. The Ethics of Philanthropy, 2012, S. 26 (28, 30).

8 Zur Unterscheidung zwischen institutionen- und individualethischer Zugangsweisen zum Problem der Weltarmut, siehe Bleisch, Pflichten auf Distanz. Weltarmut und individuelle Verantwortung, 2010, S. 16ff, 86ff.

${ }^{9}$ Eine plausible Kritik eines politischen Menschenrechtsbegriffs findet sich etwa bei Tasioulas (Fn. 6), S. 47ff.

10 Vgl. O'Neill, Tugend und Gerechtigkeit. Eine konstruktive Darstellung des praktischen Denkens, 1996, S. $170 \mathrm{ff}, 191$.
} 
bestreiten, dass es sozioökonomische Menschenrechte gibt. Sie bestreitet aber, dass sie vor und unabhängig von sozialen Institutionen bestehen können, die festlegen, gegenüber wem sie geltend gemacht werden können. Offenbar erachtet O'Neill primär den zweiten argumentativen Schritt als klärungsbedürftig. Angesichts der Tatsache, dass die Wendung ,Rechte geltend machen" (,claiming rights') zwei unterschiedliche Bedeutungsvarianten aufweist, ist es allerdings sinnvoll zu betonen, welche Bedeutung hier zugrunde gelegt wird.

Im Anschluss an Feinberg lässt sich zwischen einem performativen und einem propositionalen Geltendmachen von Rechten unterscheiden. ${ }^{11}$ Wer im ersteren Sinne ein Recht geltend macht, verändert dadurch die normative Landschaft notwendigerweise, indem etwa das Recht allererst erworben wird oder indem aufgezeigt wird, dass man das Recht tatsächlich besitzt und der Rechtsadressat entsprechend handeln muss. ${ }^{12}$ Ein klassisches Beispiel hierfür ist das Vorzeigen eines Schuldscheins. Das Geltendmachen des Rechts ist eine Voraussetzung dafür, dass der Rechtsadressat eine Zahlung vornehmen muss. Wer hingegen im propositionalen Sinne ein Recht geltend macht, verweist auf sein bestehendes Recht. Das Rechtssubjekt insistiert oder beharrt darauf, dass der Rechtsadressat handelt, wie er immer schon handeln muss. Rechte werden üblicherweise in diesem Sinne angesichts ihrer drohenden Verletzung geltend gemacht. Die Adressatin etwa des Rechts, nicht geschädigt zu werden, darf das Rechtssubjekt nicht schädigen, unabhängig davon, ob das Rechtssubjekt das Recht geltend macht. ,Ein Recht geltend machen' ist so etwas, was man mit seinem bestehenden Recht tun kann. O'Neill ist auf diese Bedeutung von ,Rechte geltend machen“ festgelegt. Das machen nicht nur ihre Beispiele, sondern auch ihre Argumentation deutlich. Entsprechend besagt der erste Schritt, dass ein Recht nur vollständig bestimmt ist, wenn klar ist, wem gegenüber das Recht besteht. Dies wiederum impliziert, dass der Einwand von O’Neill gegen die Sinnhaftigkeit des Begriffs genereller sozioökonomischer Menschenrechte schlicht besagt, dass sozioökonomische Menschenrechte nicht gegenüber jedem moralischen Akteur bestehen können.

Weshalb nun sozioökonomische Rechte nicht gegenüber jedem moralischen Akteur geltend gemacht werden können, erläutert O'Neill im Ausgang von zwei Unterschieden zwischen Freiheits- und Wohlfahrtsrechten. Der Einfachheit halber wird in diesem Unterkapitel mit dieser Terminologie gearbeitet. Der erste Unterschied lässt sich folgendermassen einführen: Freiheitsrechte müssen generell sein, wenn sie dem Rechtssubjekt einen gehaltvollen Schutz der Freiheit sichern können sollen. ${ }^{13}$ Das Recht, nicht am Betreten eines öffentlichen Platzes gehindert zu werden, ist zum Beispiel nicht viel Wert, wenn es nur gegen wenige andere gilt. ${ }^{14}$ Auch wenn O`Neill zugestehen kann, dass diese Formulierung etwas überzogen sein mag, ist der Unterschied $\mathrm{zu}$ Wohlfahrtsrechten gleichwohl offensichtlich. Hier genügt es, wenn ein Akteur das entsprechende Recht erfüllt. Der Unterschied lässt sich cum grano salis auch so formulieren, dass im Falle von Freiheitsrechten die Pflichten konjunktiv verteilt sein müssen, während sie im Falle von

\footnotetext{
${ }^{11}$ Vgl. Feinberg, The Nature and Value of Rights, in: Ders., Rights, Justice, and the Bounds of Liberty. Essays in Social Philosophy, 1980, S. 143 (149ff.) (dt. in: Stepanians (Hrsg.), Individuelle Rechte, 2007, S. 184 (192ff.)).

${ }^{12}$ Wer ein Recht in diesem Sinne geltend macht, übt eine normative Handlung aus. Vgl. von Wright, Norm and Action. A Logical Enquiry, 1963, S. 39, 75.

${ }^{13}$ Die Unterscheidung zwischen generellen und universellen Rechten findet sich allerdings bei O’Neill nicht. Das ist auch schon von Hinsch/Stepanians beanstandet worden. Vgl. Hinsch/Stepanians, Severe Poverty as a Human Rights Violation. Weak and Strong, in: Føllesdal/Pogge (Hrsg.), Real World Justice. Grounds, Principles, Human Rights, and Social Institutions, 2005, S. 295 (303/Fn. 10).

${ }^{14}$ Vgl. O’Neill (Fn. 10), S. 170f.
} 
Wohlfahrtsrechten disjunktiv verteilt sein können. ${ }^{15}$ So auffallend dieser Unterschied ist, er kann nicht zeigen, dass es keine generellen sozioökonomischen Rechte geben kann, weil sie positive Pflichten implizieren. Das lässt sich anhand eines Nothilfeszenarios zeigen, in dem ein Mann am Ertrinken ist und von mehreren zufällig anwesenden Personen gerettet werden kann. ${ }^{16}$ Es erscheint unproblematisch zu sagen, dass der ertrinkende Mann gegenüber jeder der anwesenden Personen ein Recht hat, von ihr gerettet zu werden, obwohl es genügt, wenn eine der Personen ins Wasser springt und ihn rettet. Wenn nun das schreckliche Szenario eintrifft, dass keine der anwesenden Personen ins Wasser springt und ihn rettet, erscheint es nicht minder naheliegend zu urteilen, dass jede der Personen ihre Pflicht gegenüber dem Mann, ihn zu retten, verletzt. Damit verletzt sie auch das korrelative Recht des ertrinkenden Mannes. Die Tatsache, dass keine der anderen Personen etwas hätte unternehmen müssen, wenn eine unter ihnen den Mann gerettet hätte, zeigt keineswegs, dass sie keine Pflicht ihm gegenüber hatten.

Kann es sein, dass der von O'Neill betonte Unterschied zwar nicht mit Blick auf das konstruierte Nothilfeszenario, jedoch mit Blick auf das Phänomen globaler Armut relevant ist? Tatsächlich unterscheiden sich die beiden Fälle in zentralen Hinsichten. Erstens wird im Nothilfeszenario implizit vorausgesetzt, dass jede der anwesenden Personen in der Lage ist, die ertrinkende Person zu retten. Die zweite Asymmetrie zwischen Freiheits- und Wohlfahrtsrechten, die O’Neill betont, setzt denn auch hier an. Freiheitsrechte könnten von jedermann problemlos erfüllt werden, da die Rechtsadressaten schlicht auf die Ausübung der verbotenen Handlung verzichten könnten. Hingegen scheitere der Begriff universeller und genereller Wohlfahrtsrechte daran, dass die vermeintlichen Pflichten nicht von allen erfüllt werden könnten, und sei es nur deshalb, wie O'Neill präzisiert, weil die behaupteten Rechtsadressaten körperliche Wesen seien und deshalb die entsprechenden Handlungen nicht ausüben könnten. ${ }^{17}$ Sie könnten schon gar nicht von jedem moralischen Akteur für jeden anderen Akteur ausgeübt und erfüllt werden. ${ }^{18}$ Gleichzeitig handelt es sich beim Problem von globaler Armut um ein strukturelles Problem, das nicht mit einer individuellen Handlung gelöst werden kann. Zweitens geht es im Nothilfeszenario um die Not einer einzigen Person. Im Kontext globaler Gerechtigkeit sehen sich potentielle Hilfeleister mit der Not unzähliger anderer konfrontiert. Drittens ist es im Nothilfefall klar, was konkret zu tun ist, um der notleidenden Person zu helfen. Im Falle der Weltarmut gibt es hingegen unzählige Möglichkeiten, wie die Hilfe erfolgen kann. Viertens arbeitet das Nothilfeszenario mit der Voraussetzung, dass die Kosten für die Rettung der ertrinkenden Person gering und deshalb zweifellos zumutbar sind. Diese Voraussetzung lässt sich für das Problem globaler Armut nicht ohne weiteres übernehmen. Die Kosten drohen hier für die (vermeintlichen) Rechtsadressaten sehr hoch zu werden.

Kritikerinnen und Kritiker des Begriffs genereller sozioökonomischer Menschenrechte verweisen auf mindestens einen dieser Unterschiede um zu begründen, weshalb der universellen moralischen Pflicht, Menschen zu helfen, nicht in Armut leben zu müssen kein korrelatives Recht dieser Menschen entsprechen kann. Sie gehen in der Regel davon aus, dass den Pflichten deshalb keine Rechte korrelieren können, weil die Pflichtsubjekte in der Ausübung der Pflichten einen Spielraum haben. Es handle sich um weite und deshalb unvollkommene Pflichten. Im nächsten Kapitel werden die drei meist genannten Hinsichten, in denen sich der Spielraum von unvollkommenen Pflichten äussern soll, näher in den Blick

\footnotetext{
${ }^{15}$ Vgl. auch Stepanians, O'Neill über die Notwendigkeit einer Institutionalisierung von Wohlfahrtsrechten, in: Neumaier/Sedmak/Zichy (Hrsg.), Gerechtigkeit. Auf der Suche nach einem Gleichgewicht, 2005, S. 227 (233).

${ }^{16}$ Vgl. z. B. Feinberg, Collective Responsibility, J. Phil. 65/21 (1968), S. 674 (683).

${ }^{17}$ Vgl. O'Neill, (Fn. 10), S. 170.

${ }^{18} \mathrm{Vgl}$. O'Neill, Bounds of Justice, 2000, S. 104.
} 
genommen und daraufhin untersucht, weshalb sie mit korrelativen Rechten unvereinbar sein sollen. Sie entsprechen den letzten drei der erwähnten Unterschiede zwischen Nothilfeszenarien und dem Problem globaler Armut. Nicht eigens diskutiert wird die Frage, ob die Kombination dieser drei Hinsichten ein eigenständiges Argument gegen korrelative Rechte darstellt. Der erste Unterschied wird im letzten Kapitel aufgenommen.

\section{Unvollkommene Pflichten und die These ihrer Unvereinbarkeit mit Rechten}

Um das Bedenken gegenüber dem Begriff genereller sozioökonomischer Menschenrechte angemessen erfassen zu können, hilft es, sich vor Augen zu halten, dass diesen Rechten universelle und generelle Pflichten korrelieren. Wenn jeder Mensch gegenüber jedem anderen Menschen, oder, allgemeiner, gegenüber jedem moralischen Akteur ein Recht darauf hat, dass dieser ihm hilft, nicht in Armut leben zu müssen, hat jeder moralische Akteur gegenüber jedem Menschen die Pflicht, ihm zu helfen, nicht in Armut leben zu müssen. Die erste und dritte der hier zu diskutierenden Lesarten des Spielraums von unvollkommenen Pflichten hängen direkt mit dieser Implikation des Begriffs genereller sozioökonomischer Menschenrechte zusammen.

\section{III.1 Der Spielraum hinsichtlich der Frage, wem gegenüber die Pflicht ausgeübt wird}

Wenn das gegenwärtige Ausmass globaler Armut berücksichtigt wird, ist offensichtlich, dass kein (typischer) moralischer Akteur das Recht eines jeden Menschen, nicht in Armut leben zu müssen erfüllen kann. Der moralische Akteur muss unweigerlich entscheiden, wem oder welcher Gruppe von Menschen seine Hilfe zugutekommt. Dieser Spielraum wird oft als Beleg dafür vorgebracht, dass das Pflichtsubjekt nicht jedem Menschen gegenüber die Pflicht haben kann, ihm zu helfen. ${ }^{19}$ Das Pflichtsubjekt soll vielmehr eine einzelne Pflicht haben, Menschen in Not zu helfen oder, in einer anderen Fassung, Mensch A oder B oder C zu helfen, aber nicht jedem von ihnen. Aus der Tatsache, dass ein Pflichtsubjekt entscheiden muss, wem seine Hilfe zugutekommt, folgt allerdings keineswegs, dass es nicht allen gegenüber die Pflicht haben kann, ihnen zu helfen. Es zeigt höchstens, dass es nicht allen seinen Pflichten und den ihnen korrelierenden Rechte nachkommen kann. ${ }^{20}$ Der moralische Akteur sieht sich mit einem Konflikt unzähliger Rechte und Pflichten konfrontiert. Kritiker der Sinnhaftigkeit des Begriffs genereller sozioökonomischer Rechte müssen begründen, weshalb ein solcher Konflikt begrifflich inkohärent sein soll und sich in ihr nicht schlicht der deplorable Zustand der Welt spiegelt.

Der einzige greifbare Versuch, diese Begründungspflicht tatsächlich einzulösen stammt von Simon Hope. ${ }^{21}$ Seine Begründung lässt sich als ein Argument mit drei Prämissen rekonstruieren. Die erste Prämisse besagt, dass die Nichterfüllung eines Rechts immer ein Unrecht am Rechtssubjekt darstellt. Die zweite Prämisse hält fest, dass ein (menschlicher) moralischer Akteur aufgrund der Beschränkungen der menschlichen Lebensform nicht allen seinen Pflichten nachkommen kann. Das gilt etwa für die Pflicht, Menschen in Not zu helfen. Der dritten Prämisse gemäss begeht der moralische Akteur in solchen Fällen, in denen er nicht allen seinen Pflichten nachkommen kann (oder ihnen nicht vollständig nachkommen kann), keinen moralischen Fehler. Er begeht zum Beispiel keinen Fehler, wenn er nur einigen

\footnotetext{
${ }^{19}$ Vgl. z. B. Igneski, Perfect and Imperfect Duties to Aid, Soc. Theory Pract. 32/3 (2006), S. 439 (439); Ashford (Fn. 7), S. 214.

${ }^{20}$ Vgl. Waldron, Rights in Conflict, Ethics 99/3 (1989), S. 503 (506f.).

${ }^{21}$ Vgl. Hope, Subsistence Needs, Human Rights, and Imperfect Duties, J Appl Philos 30/1 (2013), S. 88 (89, 93 , 97); Ders., Kantian Imperfect Duties and Modern Debates over Human Rights, J Polit Philos (2013), S. 1 (13f.), doi: 10.1111/jopp.12026.
} 
Menschen seine Hilfe zukommen lässt. Entsprechend tut er jenen Personen, denen er nicht hilft, weil er anderen hilft, kein Unrecht an. Aus diesen drei Prämissen schliesst Hope, dass es kein universelles Recht gegenüber allen moralischen Akteuren geben kann, dass sie dem Rechtssubjekt helfen, nicht in Armut leben zu müssen. Andernfalls würde den Menschen, denen nicht geholfen wird, ein Unrecht angetan.

Die erste Prämisse ist keineswegs unproblematisch. Hope spielt hier auf ein oft betontes Charakteristikum an, wonach die Verletzung eines Rechts nicht nur falsch ist, sondern auch ein Unrecht am Rechtssubjekt darstellt. Das darf nun allerdings nicht so verstanden werden, dass jede Nichterfüllung oder Übertretung eines Rechts falsch ist und, $a$ fortiori, ein Unrecht am Rechtssubjekt darstellt. Dies gilt nur für ungerechtfertigte Rechtsübertretungen. Gerade weil die dritte Prämisse besagt, dass der moralische Akteur unter sonst gleichen Bedingungen nicht falsch handelt, indem er die Hilfe bestimmten Menschen und nicht anderen zukommen lässt, lässt sich nicht unmittelbar schliessen, dass der Begriff genereller sozioökonomischer Menschenrechte inkohärent ist. Um dieses Argumentationsziel zu erreichen, bedürfte es der zusätzlichen Voraussetzung, dass es keine gerechtfertigten Rechtsübertretungen geben kann. Eine solche Voraussetzung wäre jedoch keineswegs trivial. Sie wäre zum Beispiel unvereinbar mit der sowohl aus dem moralischen Alltag als auch aus der Rechtsprechung und Rechtsdogmatik vertrauten Vorstellung von Rechtskonflikten. In solchen Konflikten werden die Rechte gegeneinander abgewogen und der Rechtsadressat muss entsprechend des stärksten Rechts handeln. Die schwächeren Rechte darf er gerechtfertigterweise übertreten. Rechte und Pflichten haben diesem Verständnis gemäss den Status von pro tanto-Rechten und Pflichten. Jenes Recht, das sich jeweils als das stärkste erweist, ist ,ausschlaggebend' oder ,definitiv'. ${ }^{22}$ Nur die Übertretung dieses Rechts stellt eine Rechtsverletzung dar.

Der Einwand gegen den Begriff genereller sozioökonomischer Menschenrechte greift entsprechend nur vor dem Hintergrund eines voraussetzungsreichen und kontraintuitiven Rechtsbegriffs. In der soeben vorgeschlagenen Terminologie lässt er sich so charakterisieren, dass er Rechte immer schon als ausschlaggebende Rechte versteht. Rechte sind auf der Ebene dessen verankert, was alles in allem $\mathrm{zu}$ tun richtig ist. ${ }^{23}$ Vertreter eines solchen Minderheitenmodells sind geneigt, was hier als Rechtskonflikte dargestellt wird im besten Fall als ,Scheinkonflikte“ zu verstehen, die darauf zurückzuführen sind, dass der Inhalt der Rechte noch unbestimmt ist. ${ }^{24}$

\section{III.2 Der Spielraum hinsichtlich der Frage, wie die Pflicht zu erfüllen ist}

Der zweite klassische Spielraum unvollkommener Pflichten besteht darin, dass sie in unterschiedlicher Weise und $\mathrm{zu}$ unterschiedlichen Zeitpunkten erfüllt werden können. ${ }^{25}$ Zweifellos kann die Pflicht, einem Menschen zu helfen, nicht in Armut leben zu müssen in unterschiedlicher Weise erfüllt werden. Auch ohne auf die Subtilitäten handlungstheoretischer Unterscheidungen zurückgreifen zu müssen, lässt sich zeigen, wie problematisch es ist, aus einem solchen Spielraum schliessen zu wollen, dass den Pflichten keine Rechte korrelieren können. Sowohl aus der Moralphilosophie als auch aus der Rechtswissenschaft sind durchaus Rechte vertraut, die dem Rechtsadressaten einen Spielraum hinsichtlich Art und Zeitpunkt der Erfüllung seiner Pflicht lässt. Das ist auch nicht anders zu erwarten, da diese Art von

\footnotetext{
${ }^{22}$ Vgl. Alexy (Fn. 1), S. 92, 249ff., 456.

${ }^{23}$ Auch Corinna Mieth scheint einen solchen Rechtsbegriff vorauszusetzen. Vgl. Mieth, Positive Pflichten. Über das Verhältnis von Hilfe und Gerechtigkeit in Bezug auf das Weltarmutsproblem, 2012, S. $137 \mathrm{ff}$.

${ }^{24}$ Vgl. Shafer-Landau, Specifying Absolute Rights, A. L. Rev. 37 (1995), S. 209 (210f).

${ }^{25}$ Vgl. Igneski (Fn. 19), S. 444; Mieth (Fn. 23), S. 190ff.
} 
Spielraum eine Frage von Graden ist. ${ }^{26}$ Wer aus dieser Art von Spielraum auf die begriffliche Unmöglichkeit von generellen sozioökonomischen Menschenrechten schliessen will, droht, ein gravierendes Problem falscher Negativa zu haben. Grundsätzlicher ist jedoch ein anderer Punkt. Wie O'Neill selber betont, stellen Abstraktionen ein unumgängliches Verfahren in der praktischen Philosophie dar. Durch Abstraktionen werden Prädikate , eingeklammert', die auf einen bestimmten Anwendungsbereich zutreffen, aber für die Richtigkeit oder Falschheit von Handlungen oder Behauptungen irrelevant sind. ${ }^{27}$ Es ist nun nicht einsichtig, weshalb es etwa abstrakte Prinzipien und Pflichten geben können soll, aber keine abstrakten Rechte. Ein Recht gegenüber einem moralischen Akteur, dass dieser dem Rechtssubjekt hilft, nicht in Armut leben zu müssen wird verletzt, wenn jener diese Hilfe nicht leistet. Dass er die Hilfe in verschiedenen Weisen leisten kann, ist unerheblich.

Es ist grundsätzlich denkbar, den Begriff abstrakter Pflichten und Rechte zurückweisen und beide immer schon auf der Ebene letzter Schlussfolgerungen mit Blick auf konkrete Handlungen verorten zu wollen. ${ }^{28}$ Rechte und Pflichten würden dann zum Ausdruck bringen, was in einer konkreten Situation alles in allem geboten ist. Es ist aber wichtig zu sehen, dass es sich hier um eine sehr voraussetzungsreiche und keineswegs allgemein geteilte Vorstellung handelte, im Gegenteil. Der Vorwurf, dass es sich beim Ausdruck , abstraktes Recht' um ein begriffliches Unding handelt, ist höchst kontraintuitiv.

\section{III.3 Der Spielraum hinsichtlich der Frage, wie weit der Pflicht zu folgen ist}

Der dritte Spielraum, über den unvollkommene Pflichten gerne charakterisiert werden, bezieht sich auf das Mass ihrer Erfüllung. Unvollkommene Pflichten räumen dem Pflichtsubjekt dieser Charakterisierung gemäss einen akteur-relativen Spielraum mit Blick darauf ein, wie weitgehend es einer unvollkommenen Pflicht nachkommt. ${ }^{29}$ Ein solcher Spielraum erscheint insbesondere vor dem Hintergrund der Vorstellung attraktiv, dass Akteure moralisch überfordert werden können. Diese Gefahr der moralischen Überforderung wird gerade im Kontext der globalen Armut oft betont. ${ }^{30}$ Der moralische Akteur ist hier nicht nur wie in klassischen Nothilfeszenarien ausnahmsweise gefordert. Vielmehr stellt die globale Armut eine anhaltende Herausforderung dar, der nicht mit einer einzelnen Handlung genüge getan ist. Der Kampf gegen die globale Armut hat entsprechend die Tendenz, vom moralischen Akteur beständig sehr viel zu fordern. Das Bedenken ist, dass er überfordert wird, indem er seine zentralen Interessen, Projekte und allenfalls sogar spezifischen Beziehungen opfern muss. ${ }^{31}$ Allerdings ist keineswegs klar, wie genau diesem Bedenken durch den Begriff unvollkommener Pflichten Rechnung getragen werden soll.

Im Hintergrund der These von einem akteur-relativen Spielraum des Akteurs liegt die plausible Vorstellung, dass moralische Forderungen mit den Eigeninteressen des moralischen Akteurs in Konflikt geraten können. Der These liegt, anders formuliert, die Vorstellung zugrunde, dass moralische und prudentielle Gründe konfligieren können. Der moralische Akteur hat einen Spielraum in der Frage, wie er den Konflikt löst. Grundsätzlich lassen sich zwei Lesarten dieses Spielraums unterscheiden. Die erste Lesart versteht ihn als einen aussermoralischen Spielraum: Der moralische Akteur hat aus einem aussermoralischen Gesichtspunkt einen Ermessensspielraum in der Frage, wie weit er den einschlägigen

\footnotetext{
${ }^{26}$ Vgl. Rainbolt, Perfect and Imperfect Obligations, Phil. Stud. 98/3 (2000), S. 233 (238ff).

${ }^{27}$ Vgl. O’Neill (Fn. 10), S. 59f.

${ }^{28}$ Vgl. Oberdiek, Specifying Rights Out of Necessity, O. J. L. S. 28/1 (2008), S. 127 (135, 141).

${ }^{29}$ Vgl. Meckled-Garcia, Giving Up the Goods: Rethinking the Human Right to Subsistence, Institutional Justice, and Imperfect Duties, J Appl Philos 30/1 (2013), S. 73 (80).

${ }^{30}$ Vgl. Mieth (Fn. 23), S. 203.

${ }^{31}$ Vgl. z. B. Portmore, Commonsense Consequentialism: Wherein Morality Meets Rationality, 2011, S. 25.
} 
moralischen Gründen den Vorzug vor den einschlägigen prudentiellen Gründen gibt. Diese Lesart ist für die Rekonstruktion der Unterscheidung zwischen vollkommenen und unvollkommenen Pflichten aus zwei Gründen nicht geeignet. Erstens lässt sich so die Unterscheidung zwischen vollkommenen und unvollkommenen Pflichten nicht mehr als eine innermoralische Unterscheidung verstehen. Die Diskussion über die Unterscheidung zwischen vollkommenen und unvollkommenen Pflichten verliert jedoch ihre Pointe, wenn sie nicht als eine innermoralische Unterscheidung verstanden wird. Zweitens ist diese Lesart des Spielraums zu unspezifisch. Es ist nicht einsichtig, weshalb der moralische Akteur nur mit Blick auf bestimmte Arten von Pflichten diesen Spielraum haben soll.

Der Spielraum muss denn auch als ein innermoralisch begründeter Spielraum verstanden werden. Naheliegenderweise geschieht dies in der Art, dass eine abstrakt formulierte Zumutbarkeitsgrenze in die unvollkommene Pflicht eingebaut wird: Es ist geboten, Person X zu helfen, nicht in Armut leben zu müssen, es sei denn, die Kosten für diese Hilfe sind für den moralischen Akteur zu hoch. Vollkommene Pflichten würden entsprechend keine solche Zumutbarkeitsbedingung aufweisen. Diese Art der Unterscheidung zwischen vollkommenen Pflichten, denen Rechte korrelieren und unvollkommenen Pflichten, denen keine Rechte korrelieren sieht sich jedoch mit dem Problem falscher Negativa konfrontiert. Sie macht nur Sinn, wenn vorausgesetzt wird, dass erstere nicht durch prudentielle Gründe überwogen werden können. Es ist jedoch problematisch anzunehmen, dass es überhaupt Pflichten gibt, die in diesem Sinne unabhängig von den Kosten ihrer Befolgung für die moralischen Akteure zu befolgen sind. Zwar lässt sich nicht ausschliessen, dass es bestimmte Pflichten gibt, die unter Inkaufnahme aller Kosten für das Rechtssubjekt befolgt werden müssen. $\mathrm{Ob}$ es solche Pflichten gibt und welche das wären, lässt sich allerdings nicht von vornherein bestimmen, sondern stellt sich erst nach einer Abwägung aller relevanter Faktoren heraus. Tatsächlich scheint dieser Unterscheidung zwischen vollkommenen und unvollkommenen Pflichten ein Begriff subjektiver Rechte zugrunde zu liegen, der Rechte und die korrelativen Pflichten immer schon als ausschlaggebende Rechte und Pflichten versteht. Mithin liegt auch ihr ein höchst problematischer Rechtsbegriff zugrunde. Möglicherweise ist das eine zu starke Unterstellung. Denkbar ist grundsätzlich, dass Proponenten dieser Art der Unterscheidung zwischen vollkommenen und unvollkommenen Pflichten nur die schwächere Vorstellung voraussetzen, dass Rechte immer den Ausschlag gegenüber den Eigeninteressen der Rechtssubjekte geben. Das schliesst nicht aus, dass Rechte nicht gegen andere Rechte oder allenfalls andere moralische oder nichtmoralische Erwägungen abgewogen werden können. Selbst wenn das zugestanden wird, bleibt der Einwand falscher Negativa bestehen. Mutatis mutandis greift der oben erwähnte zweite Einwand gegen die These eines aussermoralischen Spielraums mithin auch gegen diese Variante der Unterscheidung zwischen vollkommenen und unvollkommenen Pflichten.

Nicht ausgeschlossen ist durch die bisherigen Überlegungen, dass sich der Spielraum im Ausmass der Befolgung unvollkommener Pflicht in einer spezifischeren Weise rechtfertigen lässt. Einen solchen Vorschlag macht denn auch mit Immanuel Kant ein Kronzeuge der Unterscheidung zwischen unvollkommenen und unvollkommenen Pflichten:

„Allein ich soll mit einem Theil meiner Wohlfahrt ein Opfer an andere, ohne Hoffnung der Wiedervergeltung machen, weil es Pflicht ist, und nun ist unmöglich, bestimmte Grenzen anzugeben: wie weit das gehen könne. Es kommt sehr darauf an, was für jeden nach seiner Empfindungsart wahres Bedürfniß sein werde, welches zu bestimmen jedem selbst überlassen bleiben muß. Denn mit Aufopferung seiner eigenen Glückseligkeit (seiner wahren Bedürfnisse) Anderer ihre zu befördern, würde eine an sich selbst widerstreitende Maxime sein, wenn man sie zum allgemeinen Gesetz 
machte. - Also ist diese Pflicht nur eine weite; sie hat einen Spielraum, mehr oder weniger hierin zu tun, ohne daß sich die Gränzen davon bestimmt angeben lassen.“32

Der Spielraum des Pflichtsubjekts wird hier darauf zurückgeführt, dass Pflichten, welche um des guten Lebens anderer willen begründet werden, vom Pflichtsubjekt nicht in einer konsistenten Weise zugleich fordern können, sein gutes Leben aufzugeben. Auch wenn dieses Prinzip akzeptiert wird, kann es nicht leisten, was es hier leisten soll. Die Vorstellung ist zweifellos plausibel, dass die Kosten der Pflichterfüllung von den Projekten und Beziehungen, die für die Identität des Individuums und seine Vorstellungen eines geglückten Lebens konstitutiv sind, abhängen. Mithin ist es nicht unplausibel anzunehmen, dass das Ausmass der Leistungen, die von einem Subjekt um des guten Lebens anderer willen gefordert ist, individuell verschieden ist. Das impliziert jedoch lediglich, dass das Ausmass dessen, was Pflichten um des guten Lebens anderer willen konkret von einem Akteur fordern, von seinen Zielen und Plänen abhängt. Auf abstrakter Ebene lässt sich durchaus angeben, was die Pflicht fordert. Diese Form der Begründung des spezifischen Spielraums in der Erfüllung unvollkommener Pflichten scheint vorauszusetzen, dass es keine abstrakten Rechte geben kann. Auf die Kontraintuitivität einer solchen Voraussetzung ist bereits hingewiesen worden. Die Kosten eines solchen Ansatzes lassen sich mit Blick auf die von Kant evozierte Konstellation veranschaulichen. Es wäre unplausibel anzunehmen, dass die Bestimmung dessen, was im Falle von unvollkommenen Pflichten zumutbare Kosten für das Pflichtsubjekt darstellen, alleine von seiner individuellen, vormoralischen Konzeption des guten oder geglückten Lebens abhängen würde. Wenn die Kosten-Klausel gegenüber jedem inhaltlichen Lebensentwurf neutral wäre, mündete dies in eine Lesart des Spielraums, der sich nicht wesentlich von der oben zurückgewiesenen aussermoralischen unterscheiden würde. Ob der Akteur der Pflicht folgen muss oder nicht, würde vollständig von seinen moralisch kontingenten Zielen und Wünschen abhängen. Dies schriebe dem Begriff einer unvollkommenen moralischen Pflicht ein Moment der Beliebigkeit ein, das mit dem Begriff einer moralischen Pflicht nicht vereinbar scheint. Es ist nicht nur plausibler, davon auszugehen, dass der moralische Akteur mindestens teilweise eine moralische Verantwortung für seine zentralen Wünsche und Projekte besitzt, sondern auch unabhängig davon muss der moralische Akteur Handlungen auf der Basis seiner prudentiellen Interessen angesichts entgegenstehender moralischer Gründe rechtfertigen können. Es liegt nahe, dies in der Begrifflichkeit eines Konfliktes zwischen pro tanto-Rechten anderer und prudentiellen Gründen zu leisten. Wer hingegen davon ausgeht, dass vollkommene Pflichten und ihre korrelativen Rechte gegenüber prudentiellen Erwägungen begrifflich notwendig Vorrang haben, muss ein begriffliches Instrumentarium an die Hand geben um zu zeigen, wie die prudentiellen Interessen des Pflichtsubjekts gegen die moralisch relevanten Güter, die durch die vollkommenen Pflichten und Rechte geschützt werden, abzuwägen sind. In der Sprache von Rechten und Pflichten lässt sich dies diesem Ansatz gemäss nicht leisten, da diese das Resultat der Abwägung zum Ausdruck bringen.

\section{Das generelle Menschenrecht, nicht in Armut leben zu müssen}

Die bisherigen Ausführungen haben gezeigt, dass die Kritik an der Sinnhaftigkeit des Begriffs genereller sozioökonomischer Menschenrechte über die Unterscheidung zwischen vollkommenen und unvollkommenen Pflichten auf problematischen, keineswegs selbstevidenten Vorstellungen über den Begriff subjektiver Rechte und ihrer Rolle im praktischen Denken beruhen. Der Kritik liegt in unterschiedlichen Abschattungen ein revisionistisches Rechtsverständnis zugrunde, das Rechten keinen konstitutiven Platz im Rahmen von Begründungen für Handlungen und, wie zu ergänzen ist, Institutionen, einräumt,

\footnotetext{
${ }^{32}$ Kant, Metaphysik der Sitten, AA 393.
} 
sondern Rechte auf der Ebene von Schlussfolgerungen im praktischen Denken verortet. Der letzte Abschnitt versucht, diesen negativen Teil durch einen positiven Teil zu ergänzen. Einerseits werden zunächst in aller Kürze Begriff und Rolle subjektiver Rechte aus einer allgemeinen Perspektive eingeführt, andererseits wird skizziert, wie sich dies für den Begriff eines generellen Menschenrechts, nicht in Armut leben zu müssen fruchtbar machen lässt.

\section{IV.1 Rechte und gerichtete Pflichten}

Ausgehend von der Korrelativität von Rechten und Pflichten setzen Theorien subjektiver Rechte sinnvollerweise mit der Frage ein, unter welchen Bedingungen eine Pflicht gegenüber einer Entität besteht und ihr geschuldet ist und nicht nur in Bezug auf sie besteht. ${ }^{33}$ Damit wird eine Unterscheidung ins Zentrum gerückt, die in der deutschen Umgangssprache verankert ist. Nicht zuletzt dank einer berühmten Passage im Werk Kants ist ihre moralphilosophische Bedeutung vertraut, indem er eindringlich davor warnt, diese Unterscheidung zu übersehen. ${ }^{34}$ Der vielversprechendste Ansatz versteht den Unterschied zwischen diesen zwei Weisen, wie sich eine Pflicht auf eine Entität beziehen kann als einen zwischen der Begründung und dem Inhalt der Pflicht. Pflichten bestehen gegenüber einer Entität, wenn sie um ihretwillen bestehen. Sie bestehen in Bezug auf eine Entität, wenn sie inhaltlich auf sie bezogen sind. Eine Pflicht kann zugleich in Bezug auf die Entität bestehen, der gegenüber sie besteht. Diese Rekonstruktion erlaubt zu verstehen, worauf die vor allem in der Bio- und in der Naturethik zentrale Frage abzielt, welche Entitäten überhaupt Rechte haben können. Als Rechtssubjekte kommen nur Entitäten infrage, welche moralisch um ihrer selbst willen zählen. Die substantielle Frage, welche Rechte die betreffenden Rechtssubjekte haben, entscheidet sich daran, welche ihrer Eigenschaften (wie etwa Interessen) im Einzelnen Pflichten anderer begründen. Rechte bringen zum Ausdruck, welche der Eigenschaften der Rechtssubjekte um ihretwillen geschützt, gefördert oder geachtet werden sollen. Das Recht, dass andere dem Rechtssubjekt helfen, nicht in Armut leben zu müssen, kann zum Beispiel mit dem Wert für menschliche Wesen begründet werden, ein eigenständiges und selbstbestimmtes Leben führen zu können.

Ein solcher Ansatz kann auch erklären, weshalb die Verletzung gerichteter Pflichten nicht nur falsch ist, sondern ein Unrecht am Adressaten der gerichteten Pflicht und damit am Rechtssubjekt darstellt. Sie kommen nicht primär als Objekte der Pflichten, sondern als ihre Quellen in den Blick. Wenn Feinberg die zentrale moralische Bedeutung von Rechten darin sieht, dass sie erlauben, ein bestimmtes Verhalten anderer als dem Rechtssubjekt geschuldet geltend zu machen, ${ }^{35}$ hat dies damit zu tun, dass sich Rechtssubjekte als Entitäten verstehen können, die moralisch um ihrer selbst willen zählen.

Dieser Rekonstruktion gemäss bringen Rechte eine bestimmte Begründungsstruktur von Pflichten zum Ausdruck. Wie die fundamentalen und langanhaltenden Auseinandersetzungen über die Vereinbarkeit konsequentialistischer Moraltheorien mit dem Begriff subjektiver Rechte zum Ausdruck bringen, prägen Rechte denn auch die Struktur praktischen Denkens in einer entscheidenden Weise. Werden sie lediglich auf der Ebene von Schlussfolgerungen verankert, wie dies durch die Einwände gegen den Begriff genereller sozioökonomischer Rechte nahegelegt wird, kann dieser strukturbildenden Eigenheit nicht Rechnung getragen werden. Begründungen für einschlägige Handlungen erfolgten unabhängig von den jeweiligen Rechten und der spezifischen Begründungsstruktur, die sie

\footnotetext{
${ }^{33}$ Dieser und die nächsten beiden Abschnitte gehen zurück auf Schnüriger, Eine Statustheorie moralischer Rechte, 2014, S. 60ff, 163ff.

${ }^{34} \mathrm{Vgl}$. Kant (Fn. 32), AA 442.

${ }^{35}$ Vgl. Feinberg (Fn. 11), S. 148, 151.
} 
dem vorgeschlagenen Ansatz gemäss zum Ausdruck bringen.

Wie die einzelnen sozioökonomischen Rechte wie etwa das Recht, nicht in Armut leben $\mathrm{zu}$ müssen ausformuliert und individuiert werden, muss Gegenstand einer eigenständigen Untersuchung sein. Im Ausgang des zweiten Einwandes von O'Neill gegen den Begriff genereller Wohlfahrtsrechte sollen hier einzig einige Grundzüge und Implikationen solcherart begründungssensitiver genereller sozioökonomischer Menschenrechte skizziert werden.

\section{IV.2 Die teleologische Struktur sozioökonomischer Rechte}

Wie bereits erwähnt wurde, hebt O'Neills zweiter Einwand zunächst darauf ab, dass sozioökonomische Rechte nur erfüllt werden können, indem Güter zu bestimmten Zeiten und Orten geliefert werden und deshalb nicht alle Menschen in der Lage sind, die Rechte zu erfüllen. Unabhängig davon, dass eine solche Vorstellung auch unplausible Implikationen für den Begriff von generellen Freiheitsrechten hätte, gibt es keinen Grund, weshalb Rechtsadressaten ihre Pflichten nur in direktem Kontakt mit dem Rechtssubjekt erfüllen können sollen. Gewichtiger ist denn auch der weitere Punkt, dass sozioökonomische Rechte auf strukturelle Probleme verweisen, die sich - anders als dies etwa im erwähnten Nothilfeszenario der Fall ist - nicht mit einer einmaligen Handlung beheben lassen. Werden die Rechte so verstanden, dass sie den Rechtsadressaten die Pflicht auferlegen, sicherzustellen oder zu gewährleisten, dass die Rechtssubjekte nicht in Armut leben müssen oder, mit Blick auf das Recht auf Subsistenz, sicheren Zugang zu Subsistenzgütern haben, ${ }^{36}$ müssten die Adressaten genereller Rechte als durchschnittliche moralische Akteure tatsächlich zwangsläufig an dieser Aufgabe scheitern. Allerdings wurde im Rahmen dieses Beitrages bewusst darauf verzichtet, sozioökonomische Rechte begrifflich mit einer Gewährleistungspflicht zu verknüpfen. Vielmehr wurde immer auf eine Hilfspflicht rekurriert. Moralische Akteure haben die Pflicht, (anderen) Menschen zu helfen, nicht in Armut leben zu müssen. Das Recht und damit auch die korrelative Pflicht weisen zwar eine teleologische Struktur in dem Sinne auf, dass diese Hilfe auf die Realisierung eines bestimmten Zustands ausgerichtet ist. Sie impliziert aber nicht, dass die einzelnen Rechtsadressaten ihn alleine sicherstellen müssen.

Das Recht, dass andere dem Rechtssubjekt helfen, nicht in Armut leben zu müssen, stellt gleichzeitig ein abstraktes Recht dar und muss weiter konkretisiert werden. Ein vernünftiges Kriterium ist dabei, dass das Ziel bestmöglich realisiert wird. Da es offensichtlich ist, dass dies nicht möglich ist, wenn jeder Rechtsadressat für sich alleine tätig wird, besteht eine substantielle Implikation des Rechts, nicht in Armut leben zu müssen darin, dass die Rechtsadressaten gemeinsam und in einer konzertierten Weise ihren Pflichten nachzukommen suchen. ${ }^{37}$ Es erscheint allerdings nicht nötig anzunehmen, dass sozioökonomische Rechte entsprechend nicht gegenüber Individuen qua Individuen bestehen, sondern gegenüber Individuen qua Mitgliedern geeigneter Gruppen. ${ }^{38}$ Die implizite Forderung nach moralischer Arbeitsteilung nimmt hauptsächlich zwei Formen an: Weil Armut nur sinnvoll auf der Ebene politischer, wirtschaftlicher und kultureller Institutionen bekämpft werden kann, haben die Rechtssubjekte die Pflicht, sich für die Einrichtung und Ausgestaltung entsprechender Institutionen einzusetzen. Insbesondere solange angemessene Institutionen fehlen, haben sie jedoch gleichzeitig die Pflicht, in Abstimmung mit anderen

\footnotetext{
${ }^{36}$ Vgl. Meckled-Garcia (Fn. 29), S. 73f, 80.

${ }^{37}$ Vgl. Shue, Mediating Duties, Ethics 98/4 (1988), S. 687 (689ff.).

38 Vgl. Schlothfeldt, Wer ist angesichts der Weltarmut wozu verpflichtet?, in: Bleisch/Schaber (Hrsg.), Weltarmut und Ethik, 2007, S. 77 (86ff).
} 
Akteuren ihrer Hilfspflicht im Rahmen einer vorinstitutionellen moralischen Arbeitsteilung nachzukommen. ${ }^{39}$ Gleichzeitig erscheint es wenig plausibel anzunehmen, dass in Fällen, in denen selbst die Voraussetzungen für eine konzertierte Arbeitsteilung nicht gegeben sind, die einzelnen Pflichtsubjekte aus der Pflicht entlassen werden. Vielmehr haben sie im Rahmen ihrer Möglichkeiten ihrer Pflicht nachzukommen. Diese Aspekte sind im Einzelnen aus der Literatur wohlvertraut. Sie werden allerdings in der Regel vor dem Hintergrund eines alternativen Rechtsverständnisses vertreten, das etwa die dreigliedrige Struktur von Rechten aufgibt oder sie werden nicht explizit als Teil eines allgemeinen Projekts verstanden, den Kampf gegen Weltarmut und ähnliche konkrete Herausforderungen über einen einheitlichen begrifflichen Rahmen zu stützen.

Die hier vorgenommene Verteidigung des Begriffs genereller sozioökonomischer Menschenrechte erweist nicht nur, dass die Einwände gegen diesen Begriff auf einer höchst problematischen begrifflichen Basis beruhen, sondern sie legt zugleich die gemeinsame Basis sowohl institutioneller als auch nicht-institutioneller Pflichten im Kampf gegen die Armut frei. Das abstrakte generelle Menschenrecht, nicht in Armut leben zu müssen differenziert sich in eine Vielzahl konkreter Rechte, die zu unterschiedlichen Bereichen des Praktischen gehören können. So wichtig es etwa ist, zwischen individualethischen und institutionenethischen Fragen zu unterscheiden, so wichtig ist es, die Gemeinsamkeiten und Interdependenzen nicht aus dem Blick $\mathrm{zu}$ verlieren. Sie stehen nicht nur auf einem gemeinsamen substantiellen Fundament, sondern lassen sich auch mit derselben fundamentalen Begrifflichkeit rekonstruieren.

39 Eine moralische Arbeitsteilung setzt keine institutionelle Pflichtenverteilung voraus. Hinweise auf unterschiedliche Prinzipien zur Verteilung konkreter Pflichten finden sich in Cruft, Human Rights as Rights, in: Ernst/Heilinger (Hrsg.), The Philosophy of Human Rights. Contemporary Controversies, 2012, S. 129 (141). 